\title{
INVESTIGATION OF THE DYNAMICS OF A CLAMPED-CLAMPED MICROBEAM NEAR THE THIRD MODE USING A PARTIAL ELECTRODE
}

\author{
Karim M. Masri \\ Department of Mechanical \\ Engineering \\ State University of New York \\ Binghamton, NY, USA \\ kmasri1@binghamton.edu
}

\author{
Mohammad I. Younis \\ Department of Mechanical \\ Engineering \\ State University of New York \\ Binghamton, NY, USA \\ myounis@binghamton.edu \\ Physical Sciences and \\ Engineering Division \\ King Abdullah University of \\ Science and Technology \\ (KAUST), Thuwal 23955-6900, \\ Saudi Arabia \\ mohammad.younis@kaust.edu.
}

sa

\begin{abstract}
We present an investigation of the dynamics of a clampedclamped microbeam excited electrostatically near its third mode. To maximize the response at the third mode, a partial electrode configuration is utilized. A multi-mode Galerkin method is used to develop a reduced order model (ROM) of the beam. A shooting method to find the periodic motion is utilized to generate frequency response curves. The curves show hardenining behavior and dynamic pull-in. We show that the dynamic amplitude of the partial configuration is higher than that of a full electrode configuration. These results are promising for the use of higher-order modes for mass detection and for ultra sensitive resonant sensors.
\end{abstract}

\section{INTRODUCTION}

Microelectromechanical system (MEMS) are gradually developing technology. One of the important areas is MEMS resonators devices. Microresonators are used for energy harvesting [1] [2], mass sensing and detection applications [3] [4]. Ultrasensitive resonant mass sensors have been employed in various fields of science and technology, which includes environmental, chemical, biological and bacteria detection [5] [6] [7], in addition to gas identification [8] [9], and measurement of the stoichiometry of surface compounds [10].
Mass sensing is being an important topic in MEMS area. Usually Micro machined cantilevers is used for mass detection applications. There are two main ultra-sensing mass techniques. The first one is the static mode, where it measures the static bending caused by surface stresses due to analyte absorption onto a flexible cantilever coated with chemical active layer [3] [11]. The second technique is the dynamic mode, in which the microcantilever [12] [13] or the clamped-clamed [14] microbeam is oscillated at its resonance frequency. And when the beam, or the coated layer absorbs the analyte, its mass increases, so its natural frequency decreases. This shift of the natural frequency is measured, and then it can be related to the mass of the analyte.

Improving the sensitivity of micro resonators is important to obtain competitive microsensor. One way is to increase the beam width [15], it is shown that thick microcantilevers are more sensitive than thin microcantilevers for a given length. The other method is increasing the beam natural frequency [16] [15] by using light material for the microbeam, or decreasing the beam length. However, the reduction of the beam length will decrease the sensing area for the mass sensors, and also the beams higher stiffness will compromise its efficient actuation, and the movement detection may be difficult for short beams 
[15]. The third method is to actuate the resonator using higher order modes [17] [16] [18], this method has more advantages over the reduction of beam dimensions [15] [16], and it is widely proposed to enhance the sensitivity of mass microsensors.

Using higher order resonance modes needs more energy to obtain the same response achieved using the fundamental mode [19]. Usually exciting higher order modes to large motion is difficult, and the response has low amplitude and of the same range as noise. Moreover, there is a challenging of exciting asymmetric higher modes using symmetric electrostatic forces.

To overcome the above problems, we propose in this paper the use of partial electrodes that are designed specifically to maximize the response of the intended mode of vibration.

The organization of this paper is as follows. In Section 2, we present problem formulation. Section 3 we introduce the reduced order model. In section 4 Different lower electrodes configurations is presented. Section 5 shows the static response results. Section 6 the dynamic response is discussed. The main conclusion is summarized in section 7 .

\section{PROBLEM FORMULATION}

The governing equation of motion of the clamped-clamped beam [20] shown in Fig. 1, which is actuated by the electrical loads $V_{D C}$ and $V_{A C}$ applied on the beam's lower partial electrode is

$$
\begin{aligned}
& E I \frac{\partial^{4} \widehat{w}}{\partial \hat{x}^{4}}+\rho b h \frac{\partial^{2} \widehat{w}}{\partial \hat{t}^{2}}+\widehat{c} \frac{\partial \widehat{w}}{\partial \hat{x}}=\frac{E b h}{2 \ell} \frac{\partial^{2} \widehat{w}}{\partial \hat{x}^{2}} \int_{0}^{\ell}\left(\frac{\partial \widehat{w}}{\partial \hat{x}}\right)^{2} \partial \widehat{x} \\
& -\frac{\varepsilon b\left[V_{D C}+V_{A C} \operatorname{Cos}(\hat{\Omega} \hat{t})\right]^{2}\left[U\left(\widehat{x}-X_{o}\right)-U\left(\widehat{x}-X_{o}-j\right)\right]}{2(d+\widehat{w})^{2}}
\end{aligned}
$$

Where $\hat{x}$ is the position along the beam, $\hat{t}$ is time, $E$ is Young's modulus, $I$ is the moment of inertia area of the beam's cross section, $b$ is the width, $h$ is the thickness, $\ell$ is the length, $\rho$ is the density, $\hat{c}$ is the damping coefficient, $\varepsilon$ is the separation dielectric constant, $\hat{\Omega}$ is the excitation frequency, $d$ is the separation distance, and $U(\hat{x})$ is the unit step function.

The boundary conditions for a clamped-clamped beam are:

$$
\begin{array}{ll}
\widehat{w}(0, \widehat{t})=0, & \frac{\partial \widehat{w}}{\partial \widehat{x}}(0, \widehat{t})=0, \\
\widehat{w}(\ell, \widehat{t})=0, & \frac{\partial \widehat{w}}{\partial \widehat{x}}(\ell, \widehat{t})=0
\end{array}
$$

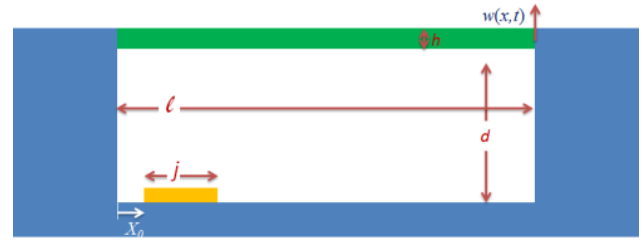

Figure 1. Clamped-Clamped beam with partial electrode.

For convenience, we introduce nondimensional variables:

$$
\begin{array}{r}
w=\frac{\widehat{w}}{d}, \quad x=\frac{\widehat{x}}{\ell}, \quad t=\frac{\widehat{t}}{T}, \\
\Omega=\widehat{\Omega T}, \quad T=\sqrt{\frac{\rho b h \ell^{4}}{E I}}
\end{array}
$$

The nondimensional governing equations and the related boundary conditions can be written as

$$
\begin{gathered}
\frac{\partial^{4} w}{\partial x^{4}}+\frac{\partial^{2} w}{\partial t^{2}}+c \frac{\partial w}{\partial x}=\alpha_{1} \frac{\partial^{2} w}{\partial x^{2}} \int_{0}^{\ell}\left(\frac{\partial w}{\partial x}\right)^{2} \partial x \\
-\frac{\alpha_{2}\left[V_{D C}+V_{A C} \operatorname{Cos}(\Omega t)\right]^{2}\left[U\left(x-X_{o}\right)-U\left(x-X_{o}-j\right)\right]}{(1+w)^{2}} \\
w(0, t)=0, \quad \frac{\partial w}{\partial x}(0, t)=0, \\
w(1, t)=0, \\
\frac{\partial w}{\partial x}(1, t)=0
\end{gathered}
$$

Where the nondimensionalization parameters are:

$$
c=\frac{\hat{c} \ell^{4}}{E I T}, \quad \alpha_{1}=6\left(\frac{d}{h}\right)^{2}, \quad \alpha_{2}=\frac{6 \varepsilon \ell^{4}}{E h^{3} d^{3}}
$$

\section{THE REDUCED ORDER MODEL}

To determine the beam response, we use the Galerkin method [20] to produce the reduced order model (ROM) of Eqn.(4) with the related boundary conditions in Eqn.(5). The beam deflection is

$$
w(x, t)=\sum_{i=1}^{n} u_{i}(t) \phi_{i}(x)
$$

Where $\phi_{i}(x)$ is the beam's ith linear undamped unforced orthonormal modeshape, and $u_{i}(t)$ is the $i$ th generalized modal coordinate. 
To solve Eqn.(5), we first multiply it by the denominator of the electrostatic force term $(1+w)^{2}$, to simplify the integration. Next, we apply the Galerkin method by substituting Eqn.(7) in Eqns.(5) and (6), multiply the resulting equation by $\phi_{j}(x)$, apply the orthonormality conditions, and integrate it over the beam domain from 0 to 1 .

$$
\begin{aligned}
& \int_{0}^{1} \phi_{j}\left(1+\sum_{i=1}^{n} u_{i} \phi_{i}\right)^{2}\left(\sum_{i=1}^{n} u_{i} \phi_{i}^{\prime \prime \prime \prime}+\sum_{i=1}^{n} \ddot{u}_{i} \phi_{i}\right) d x+c \int_{0}^{1} \phi_{j}\left(1+\sum_{i=1}^{n} u_{i} \phi_{i}\right)^{2}\left(\sum_{i=1}^{n} \dot{u}_{i} \phi_{i}\right) d x \\
& \left.=\alpha_{1} \int_{0}^{1} \phi_{j}\left(1+\sum_{i=1}^{n} u_{i} \phi_{i}\right)^{2}\left(\sum_{i=1}^{n} u_{i} \phi_{i}^{\prime \prime}\right]_{0}^{1}\left(\sum_{k=1}^{n} u_{k} \phi_{k}^{\prime}\right)^{2}\right) d x \\
& -\alpha_{2}\left[V_{D C}+V_{A C} \operatorname{Cos}(\Omega t)\right]^{2} \int_{0}^{1} \phi_{j}\left[U\left(x-X_{o}\right)-U\left(x-X_{o}-j\right)\right] d x
\end{aligned}
$$

Equation (8) is the ROM of the beam governing equation. After computing the spatial integrals, the result is a set of differential equations in terms of the modal coordinates. These Equations can be numerically integrated using Runge-Kutta technique to get $u_{i}(t)$. Then substitute the result is Eqn.(7) to estimate the total response.

\section{LOWER ELECTRODES CONFIGURATIONS}

Electrostatic force is widely used in MEMS devices. For most MEMS devices, the electrodes usually cover all the actuation area. In this section we will study the effect of the electrode's position and size of the resulting effective force.

We use different electrode configurations, one and two pieces electrodes. We vary the electrode size and position as shown in Fig.2

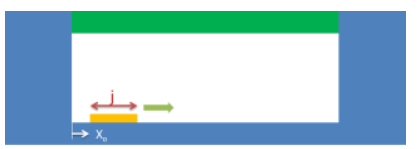

(a)

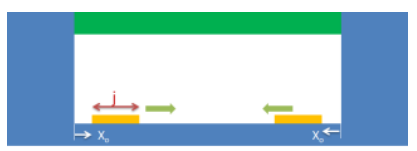

(b)
Figure 2. Clamped-clamped beam's lower electrode configurations. (a) One piece electrode. (b) Two pieces electrode.

So for the first configuration (Fig. 2a) the effective force will be

$$
\begin{aligned}
& F_{e f f}=\alpha_{2}\left[V_{D C}+V_{A C} \operatorname{Cos}(\Omega t)\right]^{2} \\
& \times \int_{0}^{1} \phi_{m}\left[U\left(x-X_{o}\right)-U\left(x-X_{o}-j\right)\right] d x
\end{aligned}
$$

where $\phi_{m}$ is the coordinate mode shape, $X_{o}$ is the distance from the starting position, and $\mathrm{j}$ is the normalized electrode size. To find the maximum force relating to each mode shape, we set the value of $\left(\alpha_{2}\left[V_{D C}+V_{A C} \operatorname{Cos}(\Omega t)\right]^{2}\right)$ to unity, and calculate the integration.
For the first mode shape, the maximum force is produced when we use a full electrode, as shown in Fig.3

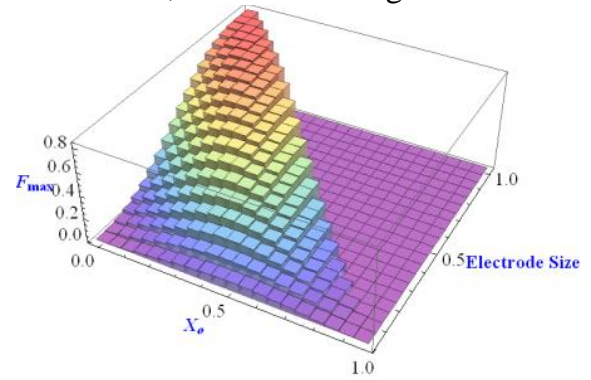

Figure 3. The maximum effective force when using the first mode shape.

For the second mode shape, the maximum results when using a half electrode $(j=1)$ either starting from ( $X_{o}=0$ or $\left.X_{o}=0.5\right)$ as shown in Fig.4.

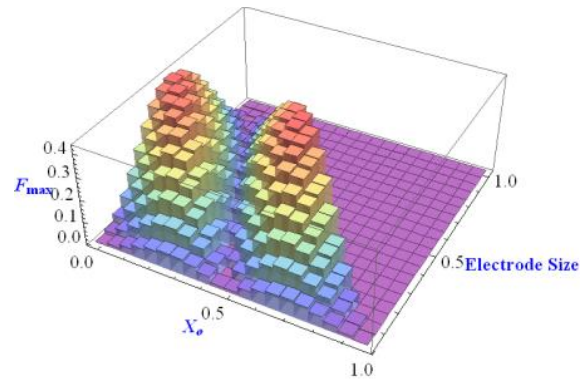

Figure 4. The maximum effective force when using the second mode shape.

On the other hand, the maximum force when using the third mode results when using the second lower beam configuration (Fig. 2b) with ( $X_{o}=0$ and $j=0.35$ ) which is using two thirds of the lower electrode full size. It is shown in Fig. 5 that using two thirds of the beams full lower electrode, almost doubles the effective force of using full lower electrode, when the third mode shape is excited.

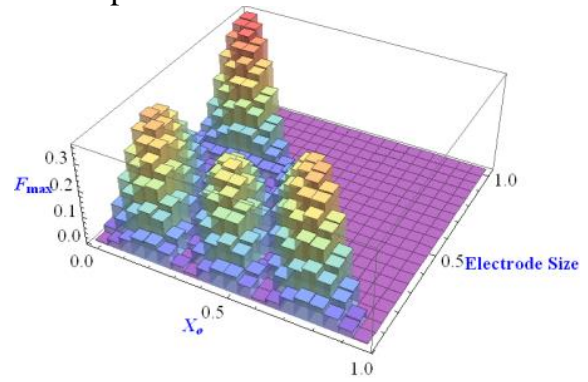

(a) 


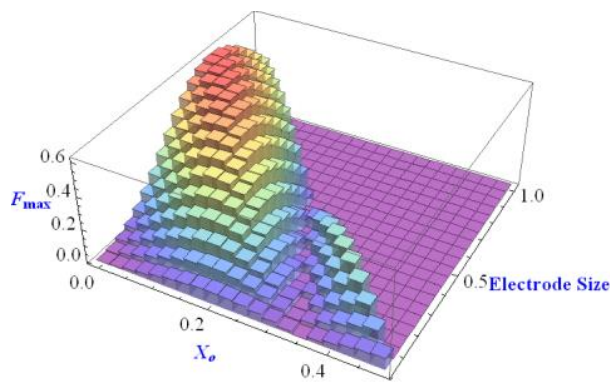

(b)

Figure 5 The maximum effective force when using the third mode shape.(a)Using 1 piece partial electrode. (b) Using 2 pieces partial electrodes.

\section{STATIC RESPONSE}

In this section of the study, we will find the effect of $V_{D C}$ on the microbeam maximum static deflection, and its pull-in voltage. To find the static response of the clamped-clamped beam, we set all the time varying terms in Eqn.(8) to zero, and the time varying modal coordinates to be unknown constants. Then we solve the nonlinear set of algebraic equation numerically to find these constants.

As a case study, we consider a clamped-clamped beam described in [21] with the following parameters, $\ell=510 \mu \mathrm{m}$, $b=100 \mu \mathrm{m}, h=1.5 \mu \mathrm{m}, d=1.18 \mu \mathrm{m}, E=166 G P a$, $\rho=2332 \mathrm{~kg} / \mathrm{m}^{3}$, and $\epsilon=8.85 \times 10^{-12} \mathrm{Fm}^{-1}$. The variation of the micro beam maximum deflection which occurs at the half of the beam $(x=0.5)$ with $V_{D C}$ is shown in Fig. 6 . The first three symmetric modeshapes are used in the ROM to simulate the maximum deflection, since it showed converged results for the response of clamped-clamped beam [22] [23] [24]. The static pull in voltage for the partial electrode is $V_{D C} \approx$ $7.6 \mathrm{~V}$ while for the full partial electrode is $V_{D C} \approx 7.6 \mathrm{~V}$.

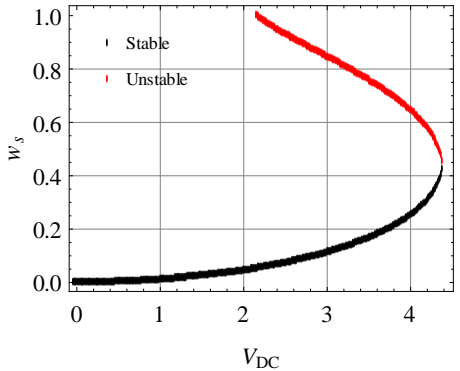

(a)

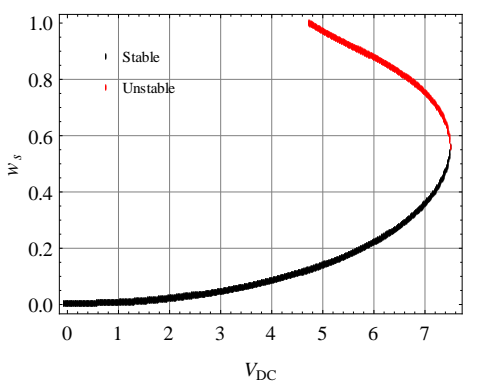

(b)

Figure 6. The beam maximum deflection with variation of $V_{D C}$. (a) Using full lower electrode. (b) Using partial lower electrode.

\section{DYNAMIC RESPONSE}

After studying the static behavior of the micro beam, now we will study the dynamic behavior of the beam when it is actuated electrostatically using partial electrodes. Fig. 7 shows the frequency response curves near the third natural frequency, simulated using the shooting method with three symmetric modes of the ROM. It shows that at the same voltage load, the partial electrode results in higher deflection than the deflection with full lower electrode.

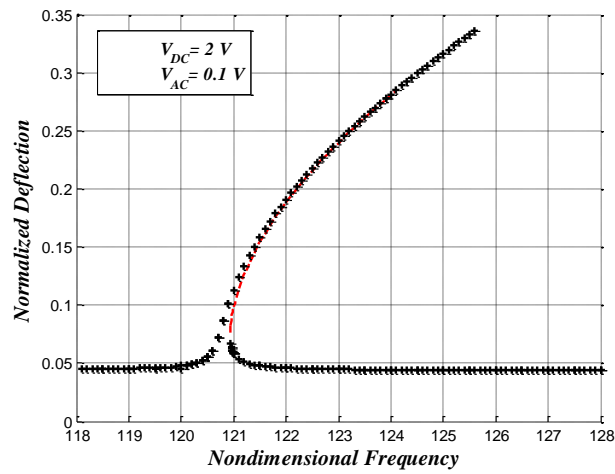

(a)

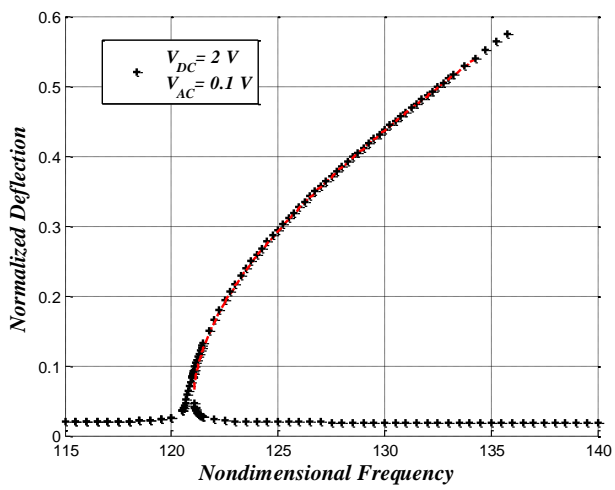

(b)

Figure 7. The Frequency response curves of clamped-clamped beam actuated with $V_{D C}=2 \mathrm{~V}$ and $V_{A C}=0.1 \mathrm{~V}$. (a) using full lower electrode, (b) using Partial lower electrode. The red dashed line is the unstable solution. 
Next, we study the effect of the actuation voltage on the beam response. Fig. 8 shows the frequency response curve for the micro beam when it is actuated with $V_{D C}=4 \mathrm{~V}$ and different $\mathrm{AC}$ voltages. We notice the hardening behavior, while the beam facing some high nonlinearity effects due to the high values of $V_{A C}$.

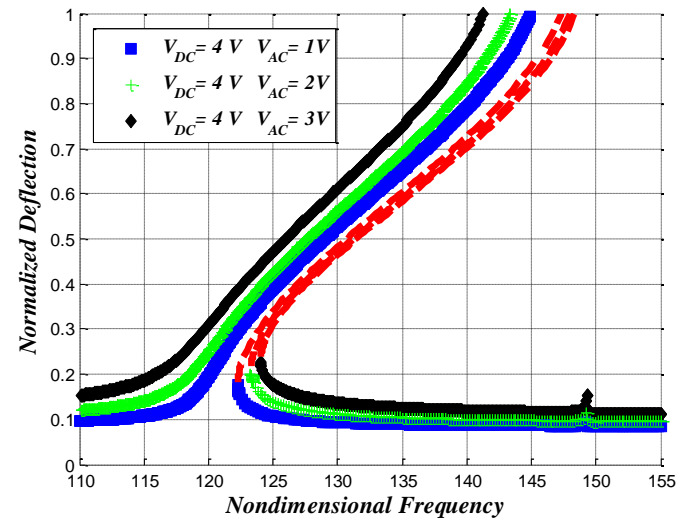

Figure 8. The Frequency response curves of clamped-clamped beam with partial electrodes when it is actuated with $V_{D C}=4 \mathrm{~V}$ and $V_{A C}=1 \mathrm{~V}, 2 \mathrm{~V}$ and $3 \mathrm{~V}$.

Fig. 9 shows the effect of higher DC voltage and the starting of a pull-in band creation, when $V_{A C}=5 \mathrm{~V}$ is used. Fig. 10 shows the frequency response curves when the micro beam is actuated with $V_{D C}=7 \mathrm{~V}$. There is a small pull-in band when $V_{A C}=2 \mathrm{~V}$ is used, and it get wider when $V_{A C}=4 \mathrm{~V}$ is used.

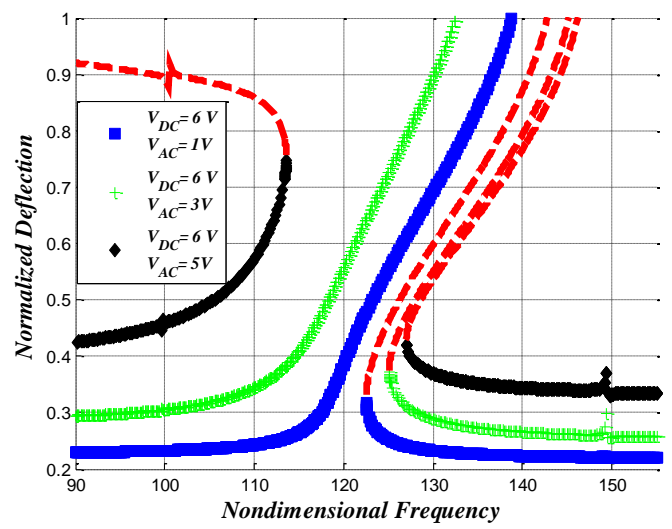

Figure 9. The Frequency response curves of clamped-clamped beam with partial electrodes when it is actuated with $V_{D C}=6 \mathrm{~V}$ and $V_{A C}=1 \mathrm{~V}, 3 \mathrm{~V}$ and $5 \mathrm{~V}$.

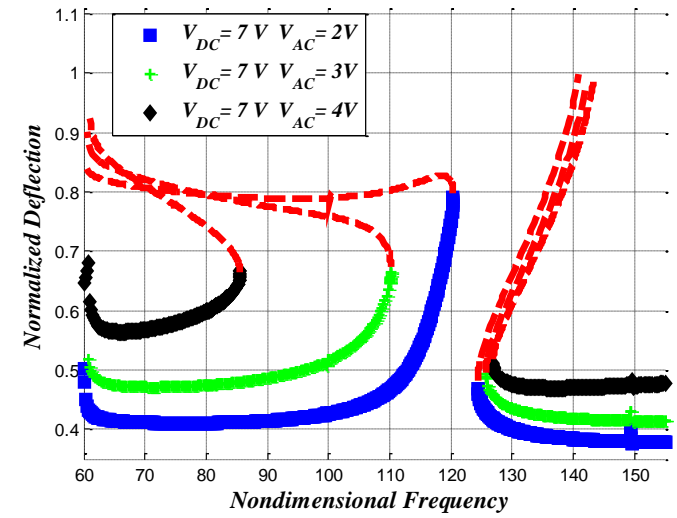

Figure 10. The Frequency response curves of clamped-clamped beam with partial electrodes when it is actuated with $V_{D C}=7 \mathrm{~V}$ and $V_{A C}=2 \mathrm{~V}, 3 \mathrm{~V}$ and $4 \mathrm{~V}$.

\section{CONCLUSIONS}

We studied the dynamics of the micro clamped-clamped beam when it is actuated with partial electrode near the third mode. We found the best configuration that provides higher response of the beam at the same actuation voltage, which is to use the first and last one-third portion of the beam as active electrodes. We used the third mode beam configuration to study the effect of the partial electrode on the static and dynamic behavior of the micro beam under the electrostatic force. We have noticed jumps, hardening behavior, and other nonlinear behaviors at higher excitation voltages. Particularly, the creation of dynamic pull-in bands and the sudden jumps due to the tangent bifurcations are promising to be utilized to improve the sensitivity of mass detection sensors or to realize MEMS switches of high operating frequencies. Experimental work is underway to verify these observed phenomena.

\section{ACKNOWLEDGMENTS}

This research has been supported by the National Science Foundation through grant 0846775.

\section{REFERENCES}

[1] Erturk, A., Inman, D.J., "A distributed parameter electromechanical model for cantilevered piezoelectric energy harvesters," Journal of Vibration and Acoustics, vol. 130, no. 4, 2008.

[2] Cottone, F., Gammaitoni, L., Vocca, H., Ferrari, M., Ferrari, V., "Piezoelectric buckled beams for random vibration energy harvesting," Smart materials and structures, vol. 21, no. 3, p. 035021, 2012.

[3] Jensenius, H., Thaysen, J., Rasmussen, A. A., Veje, L. H., Hansen, O., and Boisen A., "A microcantilever-based 
alcohol vapor sensor-application and response model," Applied Physics Letters, vol. 76, no. 18, pp. 2615-2617, 2000.

[4] Nickolay, L. V., and Datskos, P. G. , "Femtogram mass detection using photothermally actuated nanomechanical resonators," Applied Physics Letters, vol. 82, no. 16, pp. 2697-2699, 2003.

[5] Waggoner, P. S., \& Craighead, H. G. , "Micro-and nanomechanical sensors for environmental, chemical, and biological detection.," Lab on a Chip, vol. 7, no. 10, pp. 1238-1255, 2007.

[6] Ilic, B., Czaplewski, D., Craighead, H. G., Neuzil, P., Campagnolo, C., \& Batt, C., "Mechanical resonant immunospecific biological detector," Applied Physics Letters, vol. 77, no. 3, pp. 450-452, 2000.

[7] Thundat, T., Wachter, E. A., Sharp, S. L., \& Warmack, R. J. , "Detection of mercury vapor using resonating microcantilevers," Applied Physics Letters, vol. 66, no. 13, pp. 1695-1697, 1995.

[8] Maute, M., Raible, S., Prins, F. E., Kern, D. P., Weimar, U., \& Göpel, W. , "Fabrication and application of polymer coated cantilevers as gas sensors," Microelectronic engineering, vol. 46, no. 1, pp. 439-442, 1999.

[9] Kehrbusch, J., Bozek, P., Radzio, B., Ilin, E. A., \& Oesterschulze, E. , "Columnar shaped microresonators for mass detection and gas analysis.," Microelectronic Engineering, vol. 87, no. 5, pp. 817-820, 2010.

[10] Narine, S. S., \& Slavin, A. J., "se of the quartz crystal microbalance to measure the mass of submonolayer deposits: Measuring the stoichiometry of surface oxides.," Journal of Vacuum Science \& Technology A: Vacuum, Surfaces, and Films,, vol. 16, no. 3, pp. 1857-1862, 1998.

[11] Gimzewski, J. K., Gerber, Gh., Meyer, E., Schlittler, R. R., "Observation of a chemical reaction using a micromechanical sensor," Chemical Physics Letters, vol. 217, no. 5, pp. 589-594, 1994.

[12] Davis, Z. J., Abadal, G., Kuhn, O., Hansen, O., Grey, F., \& Boisen, A. , "Fabrication and characterization of nanoresonating devices for mass detection," Journal of Vacuum Science \& Technology B: Microelectronics and Nanometer Structures, vol. 18, no. 2, pp. 612-616, 2000.

[13] Kumar, V., William Boley, J. W., Yang, Y., Ekowaluyo, H., Miller, J. K., Chiu, G., Rhoads J. F., "Bifurcation-based mass sensing using piezoelectrically-actuated microcantilevers," Applied Physics Letters, vol. 98, no. 15, pp. 153510-153510-3, 2011.

[14] Younis, M. I., Alsaleem F., "Exploration of new concepts for mass detection in electrostatically-actuated structures based on nonlinear phenomena," Journal of computational and nonlinear dynamics, vol. 4, no. 2, 2009.

[15] Lochon F., Dufour I., Rebière D., "An alternative solution to improve sensitivity of resonant microcantilever chemical sensors: comparison between using high-order modes and reducing dimensions," Sensors and Actuators B: Chemical, vol. 108, no. 1, pp. 979-985, 2005.

[16] Ghatkesar, M. K., Barwich, V., Braun, T., Ramseyer, J. P., Gerber, C., Hegner, M, Lang, H. P, Drechsler, U. , Despont, M. , "Higher modes of vibration increase mass sensitivity in nanomechanical microcantilevers," Nanotechnology, vol. 18, no. 44, p. 445502, 2007.

[17] Xia, X., \& Li, X. , "Resonance-mode effect on microcantilever mass-sensing performance in air," Review of Scientific Instruments, vol. 79, no. 7, pp. 074301074301, 2008.

[18] Sharos L.B., Raman A., Crittenden S., Reifenberger R., "Enhanced mass sening using torsional and lateral," Appl. Phys. Lett., vol. 84, p. 4638, 2004.

[19] Narducci, M., Figueras, E., Lopez, M. J., Gràcia, I., Santander, J., Ivanov, P., Fonseca, L. \& Cané, C. , "Sensitivity improvement of a microcantilever based mass sensor," Microelectronic Engineering, vol. 86, no. 4, pp. 1187-1189, 2009.

[20] Younis M. I., MEMS Linear and Nonlinear Statics and Dynamics,, 1st Edition ed., Springer, 2011.

[21] Nayfeh, A. H., Younis, M. I., Abdel-Rahman E. M., "Dynamics pull-in Phenomenon in MEMS resonators," Nonlinear Dyn, vol. 48, pp. 153-163, 2007.

[22] Nayfeh, A. H., Younis, M. I., and Abdel-Rahman, E. M., "Reduced-Order Models for MEMS Applications.," Nonlinear Dyn., vol. 41, p. 211-236., 2005.

[23] Younis, M. I., Abdel-Rahman, E. M., and Nayfeh, A. H., "A Reduced-Order Model for Electrically Actuated Microbeam-Based MEMS.," J. Microelectromech. Syst., vol. 12, p. 672-680, 2003.

[24] Mohammad I Younis, Fadi Alsaleem, "Exploration of new concepts for mass detection in electrostatically-actuated structures based on nonlinear phenomena," Journal of computational and nonlinear dynamics, vol. 4, no. 2, 2009. 